\title{
A tool for pattern information extraction and defect quantification from crystal structures
}

\author{
Erhan Okuyan ${ }^{\mathrm{a}, *}$, Erkan Okuyan ${ }^{\mathrm{b}}$ \\ a TAI Inc., Ankara, Turkey \\ ${ }^{\mathrm{b}}$ Computer Engineering Department, Bilkent University, Ankara, Turkey
}

\section{A R T I C L E I N F O}

\section{Article history:}

Received 8 September 2014

Accepted 11 September 2014

Available online 25 October 2014

\section{Keywords:}

Crystallography

Material science

Pattern recognition

Primitive vectors

Basis vectors

Space group

Symmetry

Defect quantification

\begin{abstract}
A B S T R A C T
In this paper, we present a revised version of BilKristal 2.0 tool. We added defect quantification functionality to assess crystalline defects. We improved visualization capabilities by adding transparency support and runtime visibility sorting. Discovered bugs are fixed and small performance optimizations are made.
\end{abstract}

\section{New version program summary}

Program title: BilKristal 3.0

Catalogue identifier: ADYU_v3_0

Program summary URL: http://cpc.cs.qub.ac.uk/summaries/ADYU_v3_0.html

Program obtainable from: CPC Program Library, Queen's University, Belfast, N. Ireland

Licensing provisions: Standard CPC licence, http://cpc.cs.qub.ac.uk/licence/licence.html

No. of lines in distributed program, including test data, etc.: 1868923

No. of bytes in distributed program, including test data, etc.: 8854507

Distribution format: tar.gz

Programming language: C, C++, Microsoft .NET Framework 2.0 and OpenGL Libraries.

Computer: Personal Computers with Windows operating system.

Operating system: Windows XP or higher.

RAM: 20-60 Megabytes.

Classification: 8

Catalogue identifier of previous version: ADYU_v2_0

Journal reference of previous version: Comput. Phys. Comm. 185 (2014) 442

External routines: Microsoft .NET Framework 2.0. For the visualization tool, graphics card driver should also support OpenGL.

Does the new version supersede the previous version?: Yes

Nature of problem:

Determining the crystal structure parameters of a material is a very important issue in crystallography. Knowing the crystal structure parameters helps the understanding of the physical behavior of material. For complex structures, particularly for materials which also contain local symmetry as well as global symmetry, obtaining crystal parameters can be very hard.

Solution method:

The tool extracts crystal parameters such as primitive vectors, basis vectors and identifies the space group from atomic coordinates of crystal structures.

Reasons for new version:

Additional features, Compatibility issues with newer development environments, Performance optimizations, Minor bug corrections.

Summary of revisions:

- Defect quantification capability is added. The tool can process the imperfect crystal structures, finds and quantifies the crystalline defects. The tool is capable of finding positional defects, vacancy defects, substitutional impurities and interstitial impurities. The algorithms presented in [3] are used for defect quantification implementation.

\footnotetext{
* Corresponding author. Tel.: +90 5366720664.

E-mail addresses: okuyan@cs.bilkent.edu.tr (E. Okuyan), eokuyan@cs.bilkent.edu.tr (E. Okuyan).
} 
- Transparency support is added to the visualization tool. Users are now allowed to set the transparency of each atom type individually.

- Runtime visibility sorting functionality is added to facilitate correct transparency computations.

- Visual Studio 2012 support is added. Visual Studio 2012 specific project files are created and the project is tested with this development environment.

- In visualization tool, an unused log file was created. This issue is corrected.

- In visualization tool, some OpenGL calls which are executed at every draw are changed to be executed only when they are needed, improving the visualization performance.

Restrictions:

Assumptions are explained in $[1,2]$. However, none of them can be considered as a restriction onto the complexity of the problem.

Running time:

The tool was able to process input files with more than a million atoms in less than $20 \mathrm{~s}$ on a PC with an Athlon quad-core $\mathrm{CPU}$ at $3.2 \mathrm{GHz}$ using the default parameter values.

References:

[1] Erhan Okuyan, Ugur Güdükbay, Oguz Gülseren, Pattern information extraction from crystal structures, Comput. Phys. Comm. 176 (2007) 486.

[2] Erhan Okuyan, Ugur Güdükbay, BilKristal 2.0: A tool for pattern information extraction from crystal structures, Comput. Phys. Comm. 185 (2014) 442.

[3] Erhan Okuyan, Ugur Güdükbay, Ceyhun Bulutay, Karl-Heinz Heinig, MaterialVis: material visualization tool using direct volume and surface rendering techniques, J. Mol. Graphics Model. 50201450-60

(C) 2014 Published by Elsevier B.V. 